\title{
SIGNAL CONDITIONING OF CARBON NANOTUBE LOUDSPEAKER
}

\author{
A. Hall ${ }^{1 *}$, J. Gaston ${ }^{1}$, W. Wolde', S.Karna ${ }^{1}$, E. Baker ${ }^{2}$, M. Okada², Y. Wang ${ }^{2}$,and B. Farouk ${ }^{3}$ \\ 1 U.S. Army Research Laboratory, Aberdeen Proving Ground, MD, 21005 USA. \\ *Corresponding author's e-mail: asha.j.hall.civ@mail.mil \\ ${ }^{2}$ Department of Chemistry and Biochemistry, University of Maryland, College Park, MD 20742, USA \\ ${ }^{3}$ Mechanical Engineering and Mechanics Department, Drexel University, Philadelphia, PA, 19104, USA
}

Keywords: Thermophone, Carbon Nanotube Thin Film Loudspeaker, Thermoacoustics

\section{Introduction}

Acoustic phenomena can be observed when conductors are electrically heated with alternating current when either free-standing or supported by a substrate. Materials with small heat capacity per unit area posses outstanding thermoacoustic properties and can be used in thermally driven loudspeakers such as thermophones. The thermal contraction and expansion of the surrounding medium determine the amplitude of the resulting sound waves [1]. The alternate expansion and contraction of the boundary layer of air in the immediate vicinity of the thin film causes a pressure otherwise known as a sound wave. It is important to note that the sound is produced from the thermal expansion and contraction of the air in the vicinity of the thin film, and not from the mechanical movement of the thin film. However at acoustic frequencies these waves are attenuated to a small fraction of their original amplitude and at under certain operating power we observe that distortion becomes a dominate feature [2]. Other studies have recommended to add a dc offset voltage or a pulse width modulated signal to eliminate the doubling of frequency [3]. The focus of this paper is to verify their recommendation via measurements and numerical simulations of the generated thermoacoustic waves.

\section{Sample Preparations and Thermal Characterization}

The CNT film was directly pulled across two copper electrodes that serve as the points of

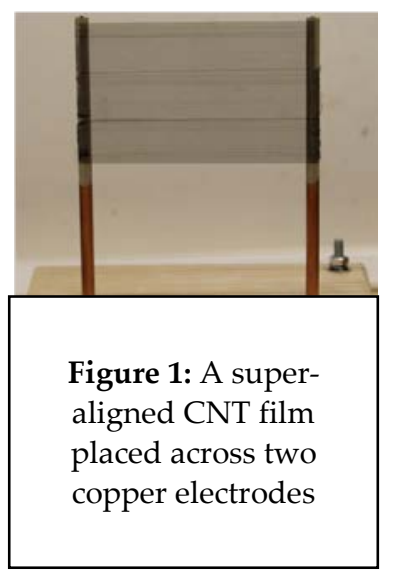
contact for the ground and current flow across the sample as shown in Figure 1. A small amount of conducting silver electrode paint (DuPont CB028 Silver Paint) was applied to the poles prior to the deposition of the thin film. The silver paint serves as an adhesive for the thin film but also improves the electrical contact between the CNT film and the copper electrode. The heat capacity and in-plane thermal conductivity of the super-aligned CNTs were determined by digital scanning calorimetry (DSC) (Perkin Elmer). The average heat capacity of the super- aligned CNT film is $490 \mathrm{~kJ} \mathrm{~kg}^{-1} \mathrm{~K}^{-1}$ and the thermal conductivity is $0.23 \mathrm{~W} / \mathrm{mK}$.

Numerical model

A fully compressible form of the Navier-Stokes equations was numerically solved to track the thermoacoustic waves generated by the CNT film heated by passing alternating current.

\section{Results and Discussion}

The CNT thin film produces a pressure oscillation in the surrounding air as it is periodically heated and cooled. This pressure oscillation leads to the generation of thermoacoustic sound 
over a wide frequency range. The experimental measurements follow a trend where the sound pressures level-off with increasing frequencies; this trend is demonstrated as a transition from the far field to the near field sound propagation as the frequency is increased. The measurements were performed in an anechoic chamber with a pure sinusoidal voltage applied to a free standing CNT thin film positioned $5 \mathrm{~cm}$ from a microphone. The output of the free-standing film reaches $90 \mathrm{~dB}$ of sound pressure at $30 \mathrm{~W}$ of power. The thermal excitation was acquired with an MIKRON TS7300 IR camera. At a power of $45 \mathrm{~W}$ and an acoustic output of $90 \mathrm{~dB}$, the maximum temperature across the surface of the free standing thin film peaked at $320^{\circ} \mathrm{F}$.

The audio frequency produced was twice the frequency of the AC current applied to the thin film speaker. This signal distortion is due to the power dissipated as being proportional to the square of the input current. In the experiment, a squared alternating current $I^{2} \sin ^{2}(\omega t)$ was supplied, wherein the Joule heating is proportional to

$$
R I^{2} \sin ^{2}(\omega t)=\frac{R I^{2}}{2}(-\cos (2 \omega t))
$$

the acoustic frequency given by $\omega=2 \pi f$. Experimental measurements confirmed that the squared sinusoidal input caused a frequency doubling in the sound pressure waveform.

Experimental test were conducted with an AC sine wave, $50 \mathrm{~V}$ dc offset, and PWM signal with an applied voltage ranging from $100-300 \mathrm{~V}$ to a free-standing CNT thin film. Preliminary tests show that when a $50 \mathrm{~V}$ dc offset was applied to the ac input voltage ranging from $50-350 \mathrm{~V}$ to the CNT thin films, the energy of the dominate tones at $2 \mathrm{kHz}$ and 4 $\mathrm{kHz}$ was equivalent. As the power increases, there is a linear increase in the energy of the dominate tones as well as the resulting harmonics.

Sample solutions from the numerical model developed are shown in Figure 2 below. A CNT thin film is sinusoidally heated with a frequency of $1 \mathrm{kHz}$, Figure 3(a). The resulting thermoacoustic pressure wave evolution $5.0 \mathrm{~cm}$ from the film is shown for early and longer times at Figures 3(b) and 3(c). The characteristic frequency doubling phenomena is predicted.
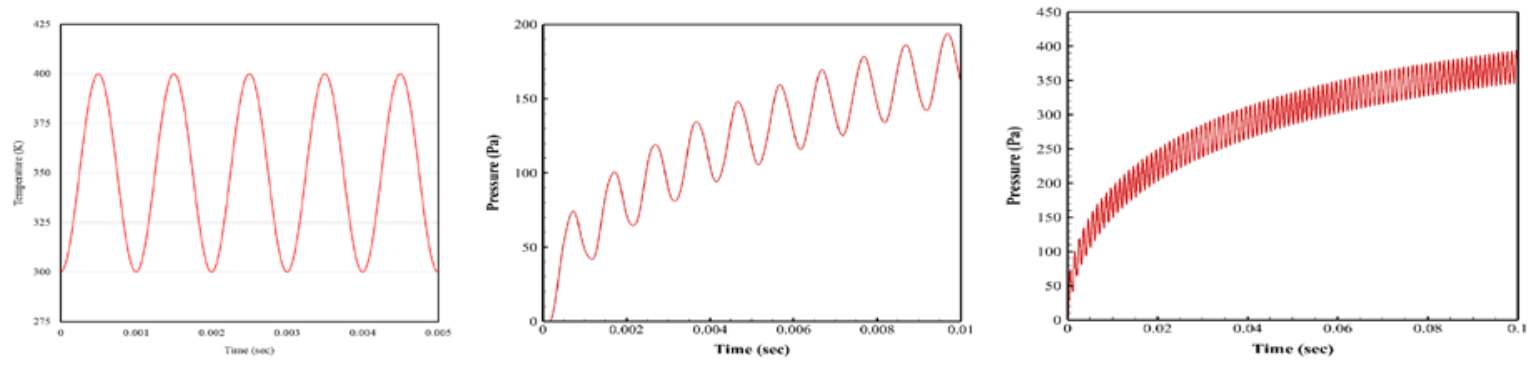

Figure 2: (a)Temperature variation of the heated film (b) predicted early time pressure variation $5.0 \mathrm{~cm}$ from the film (c) longer time pressure variation

\section{Conclusions}

The acoustic outputs consistently reached over $80 \mathrm{~dB}$. At a critical power threshold of 57W a significant amount of distortion occurred. The goal of the study is not only to eliminate distortion, but to also understand the distortion effects as a function of temperature, power, or even possible direction of air flow or trapped air surrounding the thin film.

\section{References}

[1] Arnold and Crandall, "Thermophone as a Precision Source of Sound", Phys. Rev. 10 [1] (1917) 22-38

[2] Wente, "The Thermophone", Phys Rev. 19 [4] (1922), 333-345,

[3] Aliev et al. "Thermal Transport in MWCNT sheets and yarn, Carbon 45(2007) 2880-2888.

[4] Baloch et al. "Remote Joule heating by a carbon nanotube", Nature Nanotechnology, 39, (2012),1-4

[5] Jiang et al. "Superaligned Carbon Nanotube Arrays, Films, and Yarns: A Road to Applications", Advanced Materials, 23, (2011) 1154-1161 